\title{
Isolamento social rígido durante a pandemia de COVID-19 em um estado do nordeste brasileiro
}

Rigid social isolation during COVID-19 pandemics in a state of brazilian northeast Aislamiento social rígido durante la pandemia de COVID-19 en un estado del nordeste brasileño

Ítalo Lennon Sales de Almeida ${ }^{1}$ io https://orcid.org/0000-0002-8013-8565

Thiago Santos Garces ${ }^{1}$ io https://orcid.org/0000-0002-1670-725x

George Jó Bezerra Sousa ${ }^{1}$ io https://orcid.org/0000-0003-0291-6613,

Virna Ribeiro Feitosa Cestari'1 io https://orcid.org/0000-0002-7955-0894

Raquel Sampaio Florêncio' io https://orcid.org/0000-0003-3119-7187

Thereza Maria Magalhães Moreira ${ }^{1}$ io https://orcid.org/0000-0003-1424-0649

Maria Lúcia Duarte Pereira ${ }^{1}$ io hitps://orcid.org/0000-0002-7685-6169

Rhanna Emanuela Fontenele Lima de Carvalho' io hittps://orcid.org/0000-0002-3406-9685

Almeida IL, Garces TS, Sousa GJo citar:

Florêncio RS, Moreira TM, et al. Isolamento

social rígido durante a pandemia de COVID-19 em um estado do nordeste brasileiro. Acta Paul Enferm. 2021;34:eAPE02531.

DOI

http://dx.doi.org/10.37689/actaape/2021A002531

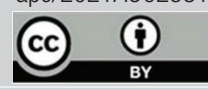

Descritores

Infeccões por coronavirus; COVID-19; Isolamento social; Unidades de terapia intensiva; Epidemiologia

Keywords

Coronavirus infections; COVID-19; Social isolation; Intensive care units; Epidemiology

Descriptores

Infecciones por coronavirus; COVID-19; Aislamiento social; Unidades de cuidados intensivos;

Epidemiología

Submetido 10 de Setembro de 2020

Aceito

2 de Dezembro de 2020

Autor correspondente

Thiago Santos Garces

E-mail: thiagogarces0812@hotmail.com

\section{Resumo}

Objetivo: Analisar a tendência temporal da incidência, mortalidade, cobertura de enfermarias e leitos de terapia intensiva e rígido isolamento social no estado do Ceará e correlacioná-los.

Métodos: Estudo ecológico, cuja variável de desfecho foi a taxa de mortalidade. Os preditores foram a incidência, a taxa de ocupação de enfermarias e leitos de terapia intensiva e a taxa de isolamento social. Foi realizada uma regressão linear múltipla considerada significativa quando $p<0,05$.

Resultados: Observou-se tendência de aumento da incidência e mortalidade por COVID-19 no estado do Ceará $(p=0,01)$. Por outro lado, observou-se tendência de diminuição na ocupação de enfermarias e leitos de terapia intensiva $(p=0,02)$. A taxa de isolamento social diminuiu significativamente durante o período $(p=$ $0,001)$. Na regressão linear múltipla, 0 isolamento social manteve-se inversamente relacionado à mortalidade pela COVID-19 ( $\beta=-0,08 ; p=0,02)$.

Conclusão: Verificou-se o efeito do rígido isolamento social durante a pandemia de COVID-19. A implementação antecipada do mesmo, com outras ações de saúde pública, mostrou-se relevante para garantir a continuidade de seus benefícios.

\section{Abstract}

Objective: This study aimed to analyze the temporal trend of incidence, mortality, coverage of wards and intensive care beds, and rigid social isolation in the Ceará State and correlate them.

Methods: Ecological study, which outcome variable was the mortality rate. Predictors were incidence, occupation rate of bed wards and intensive care beds, and social isolation rate. It was performed a multiple linear regression considering significant when $p<0.05$.

Results: It was observed an increasing trend of incidence and mortality by COVID-19 in the Ceará State $(p=0.01)$. On the other hand, it was seen a decreasing trend in the occupation of wards and intensive care beds $(p=0.02)$. The social isolation rate significantly decreased during the period $(p=0.001)$. In the multiple linear regression, social isolation remained inversely related to mortality by COVID-19 $(\beta=-0.08 ; p=0,02)$.

Conclusion: It was seen the effect of rigid social isolation during the COVID-19 pandemics. The anticipated implementation of it, with other public health actions, showed relevance to guarantee the continuity of its benefits.

\section{Resumen}

Objetivo: Analizar la tendencia temporal de la incidencia, mortalidad, ocupación de enfermerías y camas de terapia intensiva y el rígido aislamiento social en el estado de Ceará y correlacionarlos. 
Métodos: Estudio ecológico, cuya variable de criterio de valoración fue el índice de mortalidad. Los predictores fueron la incidencia, el índice de ocupación de enfermerías y camas de terapia intensiva y el índice de aislamiento social. Se realizó una regresión lineal múltiple considerada significativa cuando $p<0,05$.

Resultados: Se observó una tendencia de aumento de la incidencia y mortalidad por COVID-19 en el estado de Ceará $(p=0,01)$. Por otro lado, se observó una tendencia de reducción de ocupación de enfermerías y camas de terapia intensiva ( $p=0,02)$. El índice de aislamiento social se redujo significativamente durante el período $(p=0,001)$. En la regresión lineal múltiple, el aislamiento social se mantuvo inversamente relacionado con la mortalidad por COVID-19 $(\beta=-0,08 ; p=0,02)$.

Conclusión: Se verificó el efecto del aislamiento social rígido durante la pandemia de COVID-19. La implementación anticipada de esta medida, junto con otras acciones de salud pública, demostró ser relevante para garantizar la continuidad de sus beneficios.

\section{Introdução}

Detectado em dezembro de 2019 em Hubei-China, o novo coronavírus (SARS-CoV-2) pertence a uma família de vírus que causa doenças respiratórias em humanos. Ele se espalhou rapidamente, chamando a atençáo das autoridades de saúde chinesas e da Organizaçăo Mundial da Saúde (OMS). Logo a doença coronavírus 2019 (COVID-19) avançou para fora do território chinês, sendo caracterizada por uma pandemia pela OMS no final de janeiro, ${ }^{(1)}$ quando havia 10.000 casos confirmados na China, 80.000 casos um mês depois. No final de janeiro ${ }^{(2)}, 100$ casos foram confirmados em 19 países e 6.000 casos em 53 países um mês depois, com 750.890 casos confirmados em todo o mundo no final de março de 2020,

O Brasil confirmou seu primeiro caso $^{(3)}$ de COVID-19 em 26 de fevereiro de 2020, e um mês depois teve 4.256 casos. ${ }^{(4)}$ Logo atingiu o patamar de terceiro país em número de casos, com mais de cinco milhôes de casos e 150 mil mortes. ${ }^{(5)}$ Nesse contexto, São Paulo, Rio de Janeiro e Ceará foram os estados mais afetados no Brasil, sendo o Ceará também o estado com maior número de casos e óbitos na Região Nordeste. ${ }^{(6)}$ Acredita-se que esses números teriam sido maiores se medidas rígidas de isolamento social não tivessem sido adotadas. ${ }^{(7)}$

Neste estudo, considera-se isolamento social rígido as seguintes medidas governamentais adotadas, excepcionalmente e temporariamente no estado pesquisado: I - dever especial de reclusão; II - dever especial de proteção às pessoas do grupo de risco. III - dever especial de permanência em casa; IV - controle da circulação de veículos particulares; V-controle de entrada e saída de um município. ${ }^{(6,8)}$ Assim, em estrito isolamento social durante a pandemia de COVID-19, as pessoas permaneceram em suas residências para reduzir a transmissão da doença a grupos com maior risco de apresentar condiçóes clínicas graves. ${ }^{(9)}$ Acredita-se que essa medida tenha favorecido o achatamento da curva de casos, com a distribuiçáo dos casos por um período mais longo, permitindo uma oferta controlada e ordenada de assistência à saúde à população. Assim, a análise da evolução dos seus casos e óbitos foi fundamental para verificar o efeito das medidas tomadas. $\mathrm{O}$ aumento dos casos e da demanda por serviços de saúde é considerado uma taxa de ocupação hospitalar na clínica e Unidade de Terapia Intensiva (UTI) de fatores relevantes na análise nosológica.

Diante do exposto, o objetivo do estudo foi analisar o padráo temporal de incidência, mortalidade, cobertura de leitos hospitalares (clínicos/UTI) e estrito isolamento social no Ceará e correlacioná-los.

\section{Métodos}

Estudo ecológico realizado em 2020 no Ceará, Brasil. Todos os dados utilizados nesta pesquisa foram coletados de $1^{\circ}$ de maio a 9 de junho de 2020, pois o rígido isolamento social foi decretado nesse período. ${ }^{(6,8)}$ Tem como fonte de dados o site IntegraSUS, que agrega o banco de dados dos casos testados para COVID-19 via reaçáo em cadeia da polimerase por transcriptase reversa (RT-PCR) no estado. Esta base de dados é de domínio público, portanto, encontram-se à disposiçáo do público da Secretaria de Estado de Saúde gratuitamente. ${ }^{(10)}$

A coleta de dados foi feita por meio do preenchimento de um novo banco de dados, utilizando variáveis de interesse. A variável de desfecho foi a taxa de mortalidade diária pela COVID-19. Foi calculado pela seguinte fórmula:

Mortalidade $=100.000 \times \frac{\text { Número de óbitos por COVID-19 }}{\text { Populaçáo do Estado do Ceará }}$ 
Os preditores foram: taxa de incidência diária e taxa de ocupação de leitos de enfermarias e terapia intensiva. A incidência diária foi calculada pela fórmula:

$$
\text { Incidência }=100.000 \times \frac{\text { Número de casos novos de COVID-19 }}{\text { População do Estado do Ceará }}
$$

Além disso, a taxa de isolamento social do Ceará foi incluída por dados obtidos na plataforma Inloco, que publica um mapa brasileiro de isolamento social com base na localização do celular. ${ }^{(11)}$

A Inloco pode obter a localização com precisão através de GPS, triangulação de redes Wi-Fi, sinal bluetooth e telefonia. Ele também identifica idade, sexo, quanto tempo certos aplicativos estão abertos, operadora do usuário e modelo do telefone. A empresa criou o Índice de Isolamento Social, que permite mapear a movimentaçáo de pessoas em regióes específicas e mensurar quais apontam para maior distância social. As estatísticas geradas apontam para dados como aglomeração de pessoas e localização individual em cada região, para que seja obtido o índice de isolamento social, calculando o percentual do número absoluto de celulares rastreados. ${ }^{(11)}$

O banco de dados da Inloco conta com mais de 60 milhões de dispositivos móveis em todo o Brasil. De forma criptografada e agregada, a pesquisa permite que os órgãos responsáveis atuem diretamente nas áreas de risco ou mais afetadas pelo vírus. A plataforma também é utilizada por órgãos públicos e pela imprensa. Tal índice tem boa validade, visto que os dados gerados independem da subjetividade das respostas individuais por se tratar de dados digitais. Ressalta-se que questóes como acesso à internet, ter um celular e garantir que o indivíduo sempre mantenha um celular podem limitar o índice. ${ }^{(1)}$

A análise dos dados incluiu inicialmente a criação de linhas de tendência de cada variável. A tendência temporal foi analisada por regressão linear simples onde o preditor foi o tempo (em dias) e apresentada por meio de gráficos. Além disso, foi identificado o coeficiente determinante $\left(\mathrm{R}^{2}\right)$, que varia de 0 a 1 , onde números próximos a um identificam uma relação perfeita. Para avaliar essa tendência, foi definida como a equação linear e valor de p; o primeiro indica tendência crescente ou decrescen- te e o segundo indica se é significativa $(p<0,05)$. Quando $\mathrm{p}>0,05$, foi considerada uma tendência estacionária.

Após a análise de tendência temporal, os preditores foram relacionados ao desfecho pela correlação de Spearman. O rho $(\rho)$ de Spearman varia de $-1 \mathrm{a}+1$, onde os valores negativos indicam relaçóes inversamente proporcionais e os positivos indicam relação direta. Foram consideradas significativas as relaçóes que apresentaram $\mathrm{p}<0,05$.

Por fim, para avaliar como essas variáveis contribuem juntas para a mortalidade, foi realizada regressão linear múltipla inserindo preditores que apresentaram p <0,20 nas correlaçóes. As interpretaçôes de seus resultados são semelhantes às correlaçóes, mas com coeficientes $\beta$ que podem variar de $-\infty \mathrm{a}+\infty$. Nesse caso, $p<0,05$ também foi considerado como significância estatística. A força dessas relaçóes foi dada por seu intervalo de confiança de 95\% (IC 95\%).

Este estudo não necessita de aprovação prévia do Comitê de Ética, pois o banco de dados foi disponibilizado gratuitamente na internet pelo Governo do estado do Ceará. É importante destacar que não foi possível identificar os casos, pois não foram fornecidas informaçóes a respeito, como nome ou endereço.

\section{Results}

No primeiro dia da coleta de dados, foram notificados 273 casos e 2779 no último dia. Durante os 40 dias de análise, observou-se tendência de aumento da incidência do COVID-19 $(\mathrm{p}=0,01)$. O pico de incidência ocorreu entre 29 a 31 de maio. Em relação à mortalidade, também foi identificada como tendência crescente $(\mathrm{p}=0,01)$, sendo que 21 de maio representou o pico de óbitos no período (Tabela 1). Em relação aos preditores estudados, observou-se tendência decrescente da ocupação dos leitos das enfermarias ( $p<0,001)$ e tendência crescente da ocupaçáo dos leitos de terapia intensiva $(\mathrm{p}=0,02)$. É importante destacar que a ocupação dos leitos da enfermaria ficou acima de 100\% em dois momentos: 5 de maio (110\%) e 7 de junho 
(109\%), o que evidencia um sistema de saúde superlotado. Além disso, a taxa de isolamento social ficou em torno de $50 \%$ durante todo o período, mas diminuiu significativamente $(\mathrm{p}=0,001)$, apresentando picos (> 50\%) apenas nos domingos de maio (Tabela 1).

Tabela 1. Análise de tendência da mortalidade por COVID-19 e seus preditores

\begin{tabular}{l|cccc}
\hline Taxas & Equação linear & $\mathbf{R}^{2 a}$ & $\begin{array}{c}\text { Valor } \\
\text { de } \mathbf{p}\end{array}$ & Tendência \\
\hline Mortalidade por COVID-19 & $0,02 x-1027,3$ & 0,1352 & 0,02 & Crescente \\
$\begin{array}{l}\text { Incidência de COVID-19 } \\
\text { Ocupação de leitos na UTI }\end{array}$ & $0,49 x-21340$ & 0,1717 & 0,01 & Crescente \\
$\begin{array}{l}\text { Ocupação de leitos na } \\
\text { enfermaria }\end{array}$ & $-0,51 \mathrm{x}+224676,1$ & 0,1429 & 0,02 & Crescente \\
\hline Isolamento social & $-0,17 x+7510,6$ & 0,2672 & 0,001 & Decrescente \\
\hline
\end{tabular}

$\mathrm{a}=\mathrm{R}^{2}$ - coeficiente de determinação linear; $\mathrm{b}=$ UTI - Unidade de Terapia Intensiva

Ao correlacionar os indicadores com a mortalidade da COVID-19, evidenciou-se uma relação positiva entre a incidência da doença e sua mortalidade $(\rho=0,35 ; p=0,027)$. Também foi possível observar uma relação negativa entre a taxa de mortalidade e a taxa de ocupação das enfermarias $(\rho=-0,33 ; p=$ $0,035)$ e, principalmente, a taxa de isolamento social $(\rho=-0,57 ; p=0,001)$ (Tabela 2$)$.

Tabela 2. Correlação entre a taxa de mortalidade de COVID-19 e indicadores

\begin{tabular}{lcc}
\hline Indicadores & Rho de Spearman & Valor de $\mathbf{p}$ \\
\hline Incidência & 0,3482 & 0,027 \\
Ocupação de leitos na enfermaria & $-0,3339$ & 0,035 \\
Ocupação de leitos na UTla & $-0,0708$ & 0,664 \\
Taxa de isolamento social & $-0,5707$ & 0,001 \\
\hline
\end{tabular}

$\mathrm{a}=$ UTI - Unidade de Terapia Intensiva.

Por fim, ao ajustar as variáveis em regressão linear multivariada, evidenciou-se que a taxa de isolamento social se relacionou inversamente à mortalidade por COVID-19 $(\beta=-0,08 ; \mathrm{p}=0,02)$, demonstrando sua influência nos óbitos de a doença (Tabela 3).

Tabela 3. Regressão multivariada dos fatores relacionados à mortalidade por COVID-19

\begin{tabular}{lcccc}
\hline $\begin{array}{l}\text { Fatores relacionados à mortalidade } \\
\text { por COVID-19 }\end{array}$ & $\beta^{\mathrm{a}}$ & $\begin{array}{c}\text { Erro } \\
\text { Padrão }\end{array}$ & $\begin{array}{l}\text { Valor } \\
\text { de } \mathbf{p}\end{array}$ & IC 95\% ${ }^{\mathrm{b}}$ \\
\hline Ocupação de leitos na enfermaria & $-0,01$ & 1.30 & 0,61 & $-0,04-0,02$ \\
Incidência & 0,00 & 0,01 & 0,69 & $-0,01-0,01$ \\
Taxa de isolamento social & $-0,08$ & 0,03 & 0,02 & $-0,15--0,17$ \\
Constante & 694.91 & 194.07 & 0,001 & $300,92-1088.90$ \\
\hline
\end{tabular}

$a=\beta$ - Coeficiente da regressão linear múltipla; $b=$ IC - intervalo de confiança

\section{Discussão}

O presente estudo demonstrou o efeito do rígido isolamento social implementado na mortalidade de COVID-19. O encerramento de serviços não essenciais e a recomendação de que a população ficasse em casa, sendo permitida a circulação apenas para serviços essenciais (supermercados, farmácias e serviços de saúde) foram fundamentais para o combate ao avanço da doença. Pesquisadores previam que, com a medida do isolamento social, 1,7 milhão de vidas e os US\$ 8 trilhôes serão salvos até $1^{\circ}$ de outubro. ${ }^{(12)}$ Além disso, haverá tempo para aliviar a carga sobre os sistemas de saúde nas grandes cidades e para testar melhor os tratamentos e até mesmo a muito divulgada vacina.

A literatura científica é firme em se referir ao estrito isolamento social como ferramenta necessária para controlar a disseminação da COVID-19 e, consequentemente, as mortes pela doença. Esta pesquisa confirma essa hipótese e destaca o rígido isolamento social como estratégia positiva para o menor número de mortes no Ceará. A mortalidade por COVID-19 tem variado entre os países, influenciada pela definição não padronizada de casos, subnotificação e data do pico epidemiológico. ${ }^{(13)}$ No Brasil, os óbitos por coronavírus são significativos, ${ }^{(14)}$ o que demonstra a importância do monitoramento da doença na avaliação da gravidade e como ferramenta para a tomada de decisóes. ${ }^{(7)}$

Dados de mortalidade da doença entre as regióes do Brasil revelam que a região Nordeste é a segunda do país em número de casos, superada apenas pela região Sudeste. A maioria dos casos na região ocorre nos estados do Ceará e Bahia, ${ }^{(15)}$ com destaque para as cidades de Fortaleza e Salvador, respectivamente. Assim, a mortalidade por COVID-19 no Ceará é semelhante a outros países com muitos casos da doença. ${ }^{(7)}$

É importante ressaltar que a taxa de mortalidade pode ser afetada por casos subnotificados ou informados incorretamente; isto permitirá que as autoridades tomem as medidas necessárias e eficazes. No Brasil, a subnotificação se deve ao baixo índice de exames por milhão de habitantes. Além disso, há um atraso significativo na notificação dos resultados do teste durante as primeiras semanas do 
surto de COVID-19. Foram testados todos os casos suspeitos, bem como aqueles que estiveram em contato com um caso confirmado. No entanto, a baixa disponibilidade de testes de RT-PCR (reação em cadeia da polimerase de transcriçáo reversa) obrigou o Ministério da Saúde a recomendar o teste apenas para casos graves. Esta abordagem também foi estendida para aqueles nos grupos de alto risco (por exemplo, profissionais de saúde). ${ }^{(16)}$

Sem o intuito de reduzir a mortalidade, medidas de controle e prevenção para conter a propagação da epidemia foram tomadas pelas autoridades locais de saúde em diferentes esferas (governos federal, estadual e municipal), ${ }^{(17)}$ como práticas de higiene pessoal, exames diagnósticos e isolamento social, uma vez que a COVID-19 não é sintomática em todos os indivíduos infectados. ${ }^{(18)}$

Sem medidas de isolamento, a transmissão da doença ocorre rapidamente, causando uma curva epidêmica de aspecto acentuado, caracterizando um elevado número de casos em um curto período. Essa situação provoca uma "sobrecarga do sistema de saúde", situação de grande demanda por atendimento, sem possibilidade de resposta adequada devido ao aporte atual de capacidade instalada.

Apesar da instituição de medidas de isolamento social e estrito isolamento social, essas práticas têm causado muitas polêmicas no país, pois algumas autoridades são céticas quanto ao seu efeito. ${ }^{(17)}$ Esses dados refletem os achados deste estudo, que revelou uma taxa de isolamento social em torno de $50 \%$ e diminuindo ao longo dos dias. No mesmo período avaliado, houve queda na taxa de ocupação das enfermarias e aumento na taxa de ocupação da UTI. Esse dado pode estar relacionado à grave evolução clínica dos pacientes com a doença, além do fator tempo. O paciente que fica muito tempo internado evolui muito e é encaminhado para a UTI. Isso aumenta a ocupação da UTI e diminui a ocupação da enfermaria dependendo do pico epidemiológico.

Mesmo diante da vulnerabilidade social que a pandemia gerou, um ponto fundamental para seu enfrentamento é a diminuição da circulação de pessoas nas ruas e nos espaços públicos coletivos. ${ }^{(14)}$ Isso evita o esgotamento da capacidade dos sistemas de saúde de atender a população com formas mais gra- ves da doença, que requer internação em UTI e uso de ventilador mecânico para suporte respiratório. ${ }^{(19)}$

É visível a preocupação com a disponibilidade de leitos de UTI e ventiladores mecânicos para casos graves de internação. ${ }^{(15)}$ Vale ressaltar que, neste estudo, a taxa de ocupação da UTI não se constituiu em fator interveniente para redução da taxa de mortalidade por COVID-19 no modelo final de análise, dado este que corrobora com as evidências disponíveis, ${ }^{(20)}$ o que mostra que o aumento da disponibilidade de leitos de UTI e outros insumos para o manejo de pacientes críticos evidenciou maior efetividade à saúde pública.

Ser idoso ou ter doenças cardiovasculares, neurológicas e pulmonares tem associação significativa com o óbito pela doença, ${ }^{(7)}$ condiçóes comuns nos estados do Nordeste do Brasil. ${ }^{(21)}$ Assim, verifica-se que a taxa de ocupação da UTI é um indicador indireto da piora dos casos pela COVID-19, provavelmente devido à não adesão às medidas de isolamento social por pessoas que convivem com aquelas que apresentam fatores de risco. Também é possível que idosos ou pessoas com doenças cardiovasculares, neurológicas ou pulmonares não estejam tomando as medidas necessárias.

Apesar dos dados diários sobre a evolução da pandemia no mundo e a literatura tem mostrado uma curva ascendente para essas duas variáveis. Diante desse cenário, diversas instituiçóes de saúde têm buscado adaptar suas UTIs para o atendimento de pacientes com COVID-19. Em Bruxelas, na Bélgica, uma instituição terciária de saúde capacitou suas UTIs a estabelecer um fluxo de atendimento baseado na gestáo operacional, comunicação e apoio psicológico, além de treinar funcionários envolvidos no processo de cuidar. ${ }^{(22)}$

Mudanças na forma de cuidados e nas medidas de controle da doença são necessárias e, quando instituídas precocemente, impactam positivamente na evolução clínica. No entanto, é importante reconhecer as desigualdades sociais dentro do estado. Autores alertam que apenas uma pequena parcela da população tem acesso aos serviços de saúde, o que reafirma a relevância do rígido isolamento social. ${ }^{(7)}$

As principais limitaçóes deste estudo decorrem da utilização de dados secundários, uma vez que a subnotificação e/ou exames insuficientes influenciam na mortalidade e na taxa de incidência, limitando a visão sobre o real contexto da pandemia. 


\section{Conclusão}

$\mathrm{O}$ isolamento social rígido mostrou um efeito na redução da mortalidade durante a pandemia de COVID-19. A implantação precoce dessa ação, em conjunto com outras medidas de saúde coletiva, tem sido importante para garantir a continuidade de seus benefícios. Para manter o melhor equilíbrio possível nas medidas, os funcionários do governo devem monitorar constantemente a situação do surto e o efeito das medidas implementadas. Analisar a taxa de transmissão, o aumento diário de casos e óbitos e testar amostras representativas em diferentes contextos pode ajudar a avaliar a verdadeira prevalência da infecção. Tais informaçóes são necessárias para a tomada de decisão dos profissionais de saúde e gestores para reforçar suas diretrizes.

\section{Colaborações}

\begin{abstract}
Almeida ILS, Garces TS, Sousa GJB, Cestari VRF, Florêncio RS, Moreira TMM, Pereira MLD e Carvalho REFL contribuíram com a redação do artigo, revisão relevante do conteúdo intelectual e aprovação da versão final submetida para publicação.
\end{abstract}

\section{References}

1. World Health Organization (WHO). Coronavirus disease (COVID-19) pandemic: Situation Reports - 10. Geneva: WHO; 2020 [cited 2020 May 2]. Available from: https://www.who.int/docs/defaultsource/coronaviruse/situation-reports/20200130-sitrep-10-ncov. pdf?sfvrsn=d0b2e480_2

2. World Health Organization (WHO). Coronavirus disease (COVID-19) pandemic: Situation Reports - 11. Geneva: WHO; 2020 [cited 2020 May 2]. Available from: https://www.who.int/docs/defaultsource/coronaviruse/situation-reports/20200131-sitrep-11-ncov. pdf?sfvrsn=de7c0f7_4

3. World Health Organization (WHO). Coronavirus disease (COVID-19) pandemic: Situation Reports - 40. Geneva: WHO; 2020 [cited 2020 May 2]. Available from: https://www.who.int/docs/default-source/ coronaviruse/situation-reports/20200229-sitrep-40-covid-19. pdf?sfvrsn=849d0665_2

4. World Health Organization (WHO). Coronavirus disease (COVID-19) pandemic: Situation Reports - 71. Geneva: WHO; 2020 [cited 2020 May 2]. Available from: https://www.who.int/docs/default-source/ coronaviruse/situation-reports/20200331-sitrep-71-covid-19. pdf?sfvrsn=4360e92b_8
5. Brasil. Ministério da Saúde. Painel Corona vírus. Brasília (DF): Ministério da Saúde; 2020 [citado 2020 Mai 6]. Disponível em: https:// covid.saude.gov.br/.

6. Ceará (Estado). Decreto n. ${ }^{0} 33.574$, de 05 de maio de 2020. Governo do Estado do Ceará. [internet]. 2020. [citado 2020 Mai 12]. Disponível em: https://coronavirus.ceara.gov.br/wp-content/uploads/2020/05/ Decreto-33.574-de-05-de-maio-de-2020_DOE.pdf.

7. Sousa GJ, Garces TS, Cestari VR, Moreira TM, Florêncio RS, Pereira ML. Estimation and prediction of COVID-19 cases in Brazilian metropolises. Rev Lat Am Enfermagem. 2020;28:e3345

8. Ceará (Estado). Decreto n 33.595, de 20 de maio de 2020. Governo do Estado do Ceará. [internet]. 2020 [citado 2020 Mai 26]. Disponível em: https://www.ceara.gov.br/wp-content/uploads/2020/05/DecretosN\%C2\%BA33.595-de-20-de-maio-de-2020.pdf.

9. Werneck GL, Carvalho MS. The COVID-19 pandemic in Brazil: chronicle of a health crisis foretold. Cad Saúde Pública. 2020; 36(5):e00068820.

10. Ceará (Estado). IntegraSUS: Transparência da saúde do Ceará. Governo do Estado do Ceará. 2020 [cited 2020 Mai 28]. Disponível em: https:// integrasus.saude.ce.gov.br/.

11. Tableau public. Índice de Isolamento social Inloco, 2020 [cited 2020 may 28]. Available from: https://public.tableau.com/profile/inloco. tableau\#!/vizhome/MKTScoredeisolamentosocial/VisoGeral.

12. Zhao H, Feng Z. Staggered release policies for COVID-19 control: Costs and benefits of relaxing restrictions by age and risk. Math Biosci. 2020;326:108405.

13. Rothan HA, Byrareddy SN. The epidemiology and pathogenesis of coronavirus disease (COVID-2019) outbreak. J Autoimmun. 2020;102433.

14. Ribeiro F, Leist A. Who is going to pay the price of COVID-19? Reflections about na unequal Brazil. Int J Equ Health. 2020; 19:91-3.

15. Marinelli NP, Albuquerquer LP, Sousa IDB, Batista FM, Mascarenhas MD, Rodrigues MT. Evolution of indicators and servisse capacity at the beginning of the COVID-19 epidemic in Northeast Brazil, 2020. Epidemiol Serv Saúde. 2020; 29(3):e20200226.

16. Veiga e Silva L, de Andrade Abi Harb MD, Teixeira Barbosa dos Santos AM, de Mattos Teixeira CA, Macedo Gomes VH, Silva Cardoso EH, et al. COVID-19 Mortality Underreporting in Brazil: Analysis of Data From Government Internet Portals. J Med Internet Res. 2020;22(8):e21413.

17. Bezerra AC, Silva CE, Soares FR, Silva JA. Factor associated with people's behavior in social isolation during the COVID-19 pandemic. Cien Saúde Colet. 2020; 25(Supl.1):2411-21

18. Rothe C, Schunk M, Sothmann P, Bretzel G, Froeschl G, Wallraich C, et al. Transmission of 2019-nCoV infection from an asymptomatic contact in Germany. N Eng J Med 2020; 382:970-1.

19. Moreira RS. COVID-19: intensive care units, mechanical ventilators, and latent mortality profiles associated with case-fatality in Brazil. Cad Saúde Pública 2020; 36(5):e00080020.

20. Becher T, Frerichs I. Mortality in COVID-19 is not merely a question of resource availability. Lancet Respir Med. 2020 Sep;8(9):832-3.

21. Melo SP, Cesse EÂA, Lira PI, Rissin A, Cruz RS, Batista Filho M. [Chronic noncommunicable diseases and associated factors among adults in an impoverished urban area of the Brazilian northeast]. Cien Saude Colet. 2019;24(8):3159-3168. Portuguese.

22. Waele E, Demol J, Blockeel C, Vloeberghs V, DeVos CB, Rosseel P, Ruts L, et al. Adaptive strategies for intensive vare during the spread of COVID-19: the Brussels experience. ICU Manag Practice. 2020; 20(1):20-7. 